Research

\title{
Extension of tumor fingers: A comparison between an individual-cell based model and a measure theoretic approach
}

\author{
Marco Scianna ${ }^{1 *}$, Annachiara Colombi ${ }^{1}$ \\ ${ }^{1}$ Department of Mathematical Sciences "G. L. Lagrange", Politecnico di Torino, Corso Duca degli Abruzzi 24, \\ 10129, Torino, Italy \\ *Email address for correspondence: marco.scianna@polito.it
}

Communicated by Michele Piana and Luigi Preziosi

Received on 10 20, 2015. Accepted on 05 23, 2016.

\begin{abstract}
The invasive capability is fundamental in determining the malignancy of a solid tumor. In particular, tumor invasion fronts are characterized by different morphologies, which result both from cell-based processes (such as cell elasticity, adhesive properties and motility) and from subcellular molecular dynamics (such as growth factor internalization, ECM protein digestion and MMP secretion). Of particular relevance is the development of tumors with unstable fingered morphologies: they are in fact more aggressive and hard to be treated than smoother ones as, even if their invasive depth is limited, they are difficult to be surgically removed. The phenomenon of malignant fingering has been reproduced with several mathematical approaches. In this respect, we here present a qualitative comparison between the results obtained by an individual cell-based model (an extended version of the cellular Potts model) and by a measure-based theoretic method. In particular, we show that in both cases a fundamental role in finger extension is played by intercellular adhesive forces and taxis-like migration.
\end{abstract}

Keywords: tumor fingering, cellular Potts model, measure theoretic approach, hybrid model.

AMS subject classification: 92B05, 92C05, 92C17

\section{Introduction}

Solid tumors are thought to arise from small nodes of cells that have undergone genetic mutations and/or epigenetic alterations being therefore able to escape from DNA repair mechanisms and to cause abnormal growth regulatory mechanisms $[1,2]$. Such primary malignant colonies undergo through a relatively simple, avascular stage of growth, with nutrient and growth factor supply by diffusion from the local microenvironment $[3,4]$. However, a further search of available quantities of critical substrates results in a subsequent aggressive phase of tumor development, with the invasion of the surrounding tissue [5]. This stage of the disease is characterized by the emergence of different morphologies at the front of the malignant mass, which result both from cell-based processes (such as cell elasticity, adhesive properties and motility) and from subcellular molecular dynamics (such as growth factor internalization, ECM protein digestion and MMP secretion). Such a morphological instability determines the invasive capacity, and therefore the overall severity of the tumor. In this respect, many malignancies are characterized by the phenomenon of fingering, i.e., the formation and extension of multicellular tongues from the tumor edge, see Figure 1. In particular, malignant masses with an unstable fingered morphology are more aggressive and hard to be treated than smoother ones: in fact, even if their invasive depth is typically limited, they are difficult to be surgically removed. For instance, recent studies of photo-micrograph have shown that the "ragged" surfaces of different tumors relate to an increased severity of the disease $[6,7]$.

In this work, we indeed present two hybrid multiscale approaches, able to reproduce the fingering process of bidimensional tumor masses: an individual cell-based model deriving from a suitable version of the cellular Potts model (see for instance, [8-12]) and a theoretical framework based on the measure theory. Although obvious differences in some underlying phenomenological assumptions, the proposed approaches show a remarkable agreement in describing the phenomenon of interest and in pointing out the critical role played in finger formation (and in the overall morphological instability of the disease) of both cell-cell adhesion forces and taxis-like directional migration. 

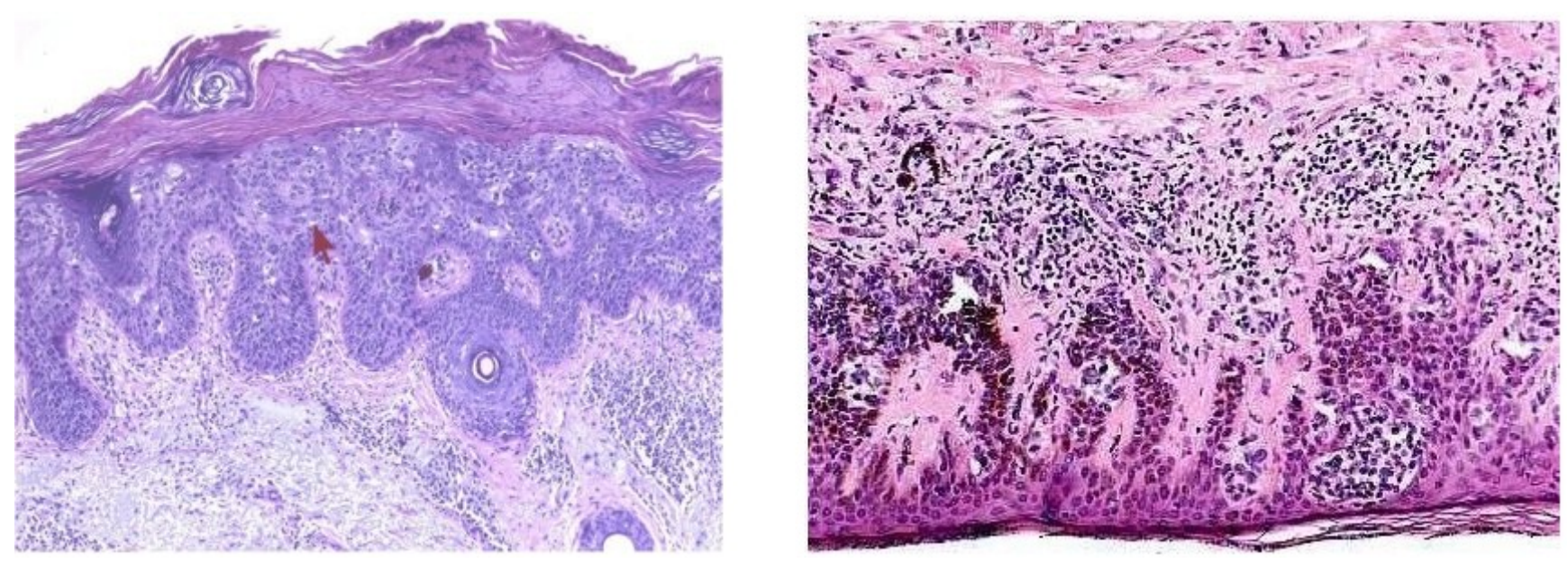

Figure 1. High-power photomicrographs of solid tumors with an unstable fingering morphology. Left panel: squamous cell carcinoma invading the mucosa membrane in the oral cavity. Right panel: a lentiginous and junctional moderate melanocytic dysplasia in the epidermis overlying the dermal component. At the bottom area of both images, it is possible to observe aggressive fronts of the malignant cells feature tentacular or finger-like extensions, similar to those reproduced by the presented computational approaches. Images courtesy of the Institute for Cancer Research and Treatment of Candiolo (I.R.C.C.).

\section{Cellular Potts model}

We first present a multilevel modeling framework for reproducing tumor fingering, which is based on the extensions of the Cellular Potts Model reviewed in [12]. In particular, the single malignant cells are represented as physical discrete objects, that locally interact with each other and with the microenvironment, whereas the biochemistry is incorporated with a macroscopic description of the evolution of ECM proteins and tumor matrix metalloproteinasis (MMPs). In this respect, we can say that the extended CPM is a hybrid-nested environment, as a discrete viewpoint is used to represent cell-scale elements, while a continuous approximation is employed to describe the molecular level.

\subsection{Model description}

The invasion of the tumor mass is modeled at the mesoscopic level using an extended version of the cellular Potts model, a grid-based stochastic approach, which realistically preserves the identity of single malignant cells and describes their behavior and interactions with the environment in energetic terms and constraints. The simulation domain is a two-dimensional regular lattice $\Omega \in \mathbb{R}^{2}$, formed by identical closed grid sites that, with an abuse of notation, will be identified by their center $\mathbf{x} \in \mathbb{R}^{2}$. Each site is labeled by an integer number, $\sigma(\mathbf{x}) \in \mathbb{N}$, that can be interpreted as a degenerate spin originally coming from statistical physics $[13,14]$. As classically adopted in CPMs, a neighboring site of $\mathbf{x}$ is denoted by $\mathbf{x}^{\prime}$, while its overall neighborhood by $\Omega_{\mathbf{x}}^{\prime}$, i.e., $\Omega_{\mathbf{x}}^{\prime}=\left\{\mathbf{x}^{\prime} \in \Omega: \mathbf{x}^{\prime}\right.$ is a neighbor of $\left.\mathbf{x}\right\}$. Each tumor cell is identified by $\Sigma_{\sigma}$ and consists of a subdomain of contiguous sites with identical spin (i.e., $\Sigma_{\bar{\sigma}}=\{\mathbf{x} \in \Omega: \sigma(\mathbf{x})=\bar{\sigma}\}$ with $\bar{\sigma}=1, \ldots, N(t)$, where $N(t)$ is the total number of individuals at time $t$ ), and has an associated type $\tau\left(\Sigma_{\sigma}\right)=C$. The tumor mass resides in an extracellular matrix which, as classically done in these types of model [12,15-17], is represented as a further special, generalized cell $\Sigma_{\sigma=0}$ of type $\tau=M$ : it is assumed to be passive, isotropically distributed throughout the simulation domain, forming no large-scale structures, as it is composed of a mixture of soluble components (among others, long carbohydrate polymers, and non-proteoglycan polysaccharides) together with the water solvent.

The malignant cells move and behave to iteratively and stochastically reduce the free energy of the overall system, given by an hamiltonian $H$, whose expression will be clarified below. The core algorithm consists of elementary steps of a modified Metropolis method for Monte Carlo-Boltzmann dynamics $[11,18]$. This approach is particularly suitable to implement the natural exploratory behavior of biological 


\section{Scianna, A. Colombi}

individuals. Procedurally, at each time step $t$, called Monte Carlo Step (MCS, the basic unit of time of the model), a randomly selected lattice site $\mathbf{x}_{\mathrm{s}}$ (s for source) attempts to copy its spin, $\sigma\left(\mathbf{x}_{\mathrm{s}}\right)$, into one of its unlike neighbors, $\mathbf{x}_{\mathrm{t}} \in \Omega_{\mathbf{x}_{\mathrm{s}}}^{\prime}: \mathbf{x}_{\mathrm{t}} \notin \Sigma_{\sigma\left(\mathbf{x}_{\mathrm{s}}\right)}$ (t for target) which is also randomly selected. In particular, if $\tau\left(\Sigma_{\sigma\left(\mathbf{x}_{\mathrm{s}}\right)}\right)=C$ (i.e., if $\left.\sigma\left(\mathbf{x}_{\mathrm{s}}\right)=1, \ldots, N(t)\right)$, the cell $\Sigma_{\sigma\left(\mathbf{x}_{\mathrm{s}}\right)}$ is protruding (i.e., extending its filopods towards another cell or in the extracellular space). Otherwise, if $\tau\left(\Sigma_{\sigma\left(\mathbf{x}_{\mathbf{s}}\right)}\right)=M$ (i.e., if $\left.\sigma\left(\mathbf{x}_{\mathbf{s}}\right)=0\right)$, the cell $\Sigma_{\sigma\left(\mathbf{x}_{\mathrm{t}}\right)}$ is retracting. Each trial spin update is accepted with a Boltzmann-like probability function $P\left(\sigma\left(\mathbf{x}_{\mathrm{s}}\right) \rightarrow \sigma\left(\mathbf{x}_{\mathrm{t}}\right)\right)$ :

$$
P\left(\sigma\left(\mathbf{x}_{\mathrm{s}}\right) \rightarrow \sigma\left(\mathbf{x}_{\mathrm{t}}\right)\right)(t)=\min \left\{1, \exp \left(\frac{-\Delta H}{T_{C}}\right)\right\},
$$

where $\Delta H$ is the net difference of the hamiltonian due to the proposed change of domain configuration and $T_{C}$ is a Boltzmann temperature, that measures the intrinsic cell motility. We here set a Boltzmann temperature constant and common for all cells: however, it may be a distinct property of each malignant individual, as it may be related to its biochemical state (for a detailed comment, see [12]).

For any given time $t$ the free energy of the system, whose minimization drives its evolution, is:

$$
H(t)=H_{\text {shape }}(t)+H_{\text {adhesion }}(t)+H_{\text {haptotaxis }}(t) .
$$

$H_{\text {shape }}$ models the cell geometrical attributes, which are written as relative deformations in the following form: (see [12] for a more detailed explanation):

$$
\begin{aligned}
H_{\text {shape }}(t) & =H_{\text {surface }}(t)+H_{\text {perimeter }}(t) \\
& =\sum_{\Sigma_{\sigma}}\left[\kappa_{C}\left(\frac{s_{\Sigma_{\sigma}}(t)-S_{C}}{s_{\Sigma_{\sigma}}(t)}\right)^{2}+\nu_{C}\left(\frac{p_{\Sigma_{\sigma}}(t)-P_{C}}{p_{\Sigma_{\sigma}}(t)}\right)^{2}\right],
\end{aligned}
$$

which depend on their actual surface and perimeter, $s_{\Sigma_{\sigma}}(t)$ and $p_{\Sigma_{\sigma}}(t)$, as well as on the same quantities in the relaxed state, $S_{C}$ and $P_{C}$, which correspond instead to the mean measures of tumor cells in resting conditions. $\kappa_{C}$ and $\nu_{C} \in \mathbb{R}_{+}$are mechanical moduli in units of energy: in particular, $\kappa_{C}$ refers to cell surface changes, while $\nu_{C}$ relates to cell deformability/elasticity, i.e., the ease with which an individual is able to remodel changing its perimeter. Assuming that the cells do not significantly grow during invasion, the fluctuations of their volumes are kept negligible with $\kappa_{C} \gg 1$. Moreover, because tumor cells are typically deformable, as they are able to significantly remodel to invade their surroundings more efficiently, we set $\nu_{C}<1$.

$H_{\text {adhesion}}$, deriving from the Steinberg's Differential Adhesion Hypothesis (DAH) [11,19], measures the adhesive interactions between different cells or between a cell and Matrigel elements:

$$
H_{\text {adhesion }}(t)=\sum_{\mathbf{x} \in \Sigma_{\sigma}, \mathbf{x}^{\prime} \in \Sigma_{\sigma^{\prime}}} J_{\tau\left(\Sigma_{\sigma(\mathbf{x})}\right), \tau\left(\Sigma_{\sigma^{\prime}\left(\mathbf{x}^{\prime}\right)}\right)},
$$

where $\mathbf{x}$ and $\mathbf{x}^{\prime}$ are two neighboring sites while $\Sigma_{\sigma}$ and $\Sigma_{\sigma^{\prime}}$, where $\sigma \neq \sigma^{\prime}$, two neighboring elements. The coefficients $J_{\tau\left(\Sigma_{\sigma}\right), \tau\left(\Sigma_{\sigma^{\prime}}\right)} \in \mathbb{R}_{+}$are binding forces per unit area and are obviously symmetric w.r.t. the indices. In particular, $J_{C, C}$ represents the adhesive strength between the membranes of two nearby tumor cells, a measure of the quantity of active and exposed cadherins. $J_{C, M}$ evaluates instead the heterotypic adhesive bonds between the integrins on the cell surface and suitable ligands present in the extracellular matrix. Since malignant cells have been demonstrated to have reduced cell-cell adhesiveness but increased cell-ECM adhesiveness, due to a change in the relative expression of the corresponding adhesive molecules [20], we set $J_{C, M}=2 J_{C, C}$.

$H_{\text {haptotaxis }}$ reproduces the effect of cell preferential movement in the direction of zones with higher concentration of extracellular matrix proteins (such as fibrin, vitronectin, and some of the collagen family) and is implemented with a local linear-type relation:

$$
\Delta H_{\text {haptotaxis }}=\mu\left[p_{t}\left(\mathbf{x}_{\mathrm{t}}, t\right)-p_{t}\left(\mathbf{x}_{\mathrm{s}}, t\right)\right],
$$


where $\mathbf{x}_{\mathrm{S}}$ and $\mathbf{x}_{\mathrm{t}}$ are, respectively, the source and the final lattice site randomly selected during a trial update in a MCS, see (1). $p_{t}(\mathbf{x}, t)=p(\mathbf{x}, t)+\sum_{\mathbf{x}^{\prime} \in \Omega_{\mathbf{x}}^{\prime}} p\left(\mathbf{x}^{\prime}, t\right)$, where $\mathbf{x} \in\left\{\mathbf{x}_{\mathbf{S}}, \mathbf{x}_{\mathrm{t}}\right\}$, evaluates the local level of ECM proteins sensed by the moving cell membrane site, as $p(\mathbf{x}, t)$ is their amount at site $\mathbf{x}$ (defined in Equation (7)). Finally, $\mu \in \mathbb{R}^{+}$represents the local strength of haptotaxis. The term (5) is similar to that used in [21] for the chemotactic processes in Dictyostelium Discoideum aggregation.

Malignant cells are also allowed to proliferate. In particular, the time between successive cell divisions is assumed to have a stochastic distribution, which depends on the time interval from last mitotic process (i.e., except for extremely rapidly dividing cells, whose likelihood of reentering the $\mathrm{S}$ phase is extremely small, see [1]). We therefore define the present probability $P_{\Sigma_{\sigma}}$ for cell $\Sigma_{\sigma}$ to undergo mitosis with the following functional form, which resembles that used in similar approaches [17]:

$$
P_{\Sigma_{\sigma}}(t)= \begin{cases}0, & \text { if }\left(t-t_{\Sigma_{\sigma}}\right) \leq t_{0} \\ \frac{\left(t-t_{\Sigma_{\sigma}}\right)^{2}}{1+\left(t-t_{\Sigma_{\sigma}}\right)^{2}}, & \text { if }\left(t-t_{\Sigma_{\sigma}}\right)>t_{0} .\end{cases}
$$

$t_{\Sigma_{\sigma}}$ is the last time that cell $\Sigma_{\sigma}$ underwent mitosis, while $t_{0}$ is a dormant period during which cells are prohibited to proliferate (i.e., it corresponds to the G1 phase, during which cells are metabolically active and grow). Procedurally, the mechanism of cell division is implemented by dividing the proliferating individual into two identical daughter cells with a halved area with respect to their parent: however, due to the shape constraints in Equation (3), they will gradually "maturate" into full-size cells. We further assume that both daughter cells evenly inherit all the parent's biophysical properties. Finally, the newly formed individuals are randomly placed around the parent cell center of mass. In particular, $t_{0}$ is set equal to $1300 \mathrm{MCS}$ (i.e., $\approx 7$ hours): since the mitotic rate varies greatly according to the specific tissue and type of malignancy, this choice represents a compromise between very slow growing and very aggressive tumor types.

The matrix substrate is assumed to initially contain saturating levels of soluble proteins, that can naturally decay and be degraded by the metalloproteinases (MMPs) secreted by malignant cells. The change in the local amount of ECM proteins is denoted by $p(\mathbf{x}, t)$ and described by:

$$
\frac{\partial p(\mathbf{x}, t)}{\partial t}=-\underbrace{\lambda_{p} p(\mathbf{x}, t)}_{\text {decay }}-\underbrace{\chi_{p} p(\mathbf{x}, t) m(\mathbf{x}, t)}_{\text {degradation }},
$$

where we set $\lambda_{p} \ll \chi_{p}$ to indicate a much higher dissolution of ECM proteins due to the activity of cell proteolytic enzymes than with respect to the physiological decay. The concentration of tumor-secreted MMPs, indicated by $m(\mathbf{x}, t)$, is governed by:

$$
\frac{\partial m(\mathbf{x}, t)}{\partial t}=\underbrace{D_{m} \nabla^{2} m(\mathbf{x}, t)}_{\text {diffusion }}-\underbrace{\lambda_{m} m(\mathbf{x}, t) \delta_{\tau\left(\Sigma_{\sigma(\mathbf{x})}\right), M}}_{\text {decay }}+\underbrace{\pi_{m} \delta_{\tau\left(\Sigma_{\sigma(\mathbf{x})}\right), C}}_{\text {production }},
$$

where $\lambda_{m}$ is the decay rate in the ECM and $D_{m}$ the diffusion coefficient, whose low value models the fact that proteolysis is strongly localized in the regions close to the cell membrane, in agreement with experimental evidence in [22]. Finally, $\pi_{m}$ is the constant production rate of degrading enzymes from malignant cells (i.e., we suppose that initially there are not MMPs dissolved in the matrix).

The coupling between the reaction-diffusion equations for the microenvironmental variables and the CPM discrete dynamics is achieved at the numerical level. Procedurally, the grid for the numerical solution of each PDE is in fact matched with the CPM lattice and, at every time step, each computational module is used as the initial condition for the others. In particular, the main lines of the computational algorithm are: (i) the discrete CPM evolves through an MCS, following the standard Metropolis rule; (ii) the local quantities of the chemical variables are computed, based on the new cell configuration (in particular, after the spin flip, the target site, $\mathbf{x}_{\mathrm{t}}$, is assigned the same concentrations of the microscopic variables as 


\section{Scianna, A. Colombi}

the moving source site, $\mathbf{x}_{\mathrm{s}}$ ); (iii) the continuous equations of the fields are rederived, according to the new distribution of the continuous variables themselves and to the new boundaries of the simulated discrete objects, and solved using a finite element scheme, characterized by 30 diffusion time steps per MCS (this temporal step is sufficiently small to guarantee numerical stability); (iv) the biophysical properties of each discrete individual (determined by its Potts coefficients) are updated, given its new intracellular state and the new configuration of the lattice; (v) the hamiltonian functional of the system is updated, and the system is ready to evolve again.

Table 1. Parameter setting of the CPM simulations

\begin{tabular}{|c|c|c|}
\hline \hline Parameter & Description & Model Value \\
\hline \hline$S_{C}$ & mean cell surface & $200\left[\mu \mathrm{m}^{2}\right]$ \\
$P_{C}$ & mean cell perimeter & $60[\mu \mathrm{m}]$ \\
$T_{C}$ & cell motility & 2.5 \\
$\kappa_{C}$ & cell volume compressibility & 5 \\
$\nu_{C}$ & cell elasticity & 0.5 \\
$\nu_{N}$ & nucleus elasticity & 10 \\
$J_{C, C}$ & cell-cell adhesive strength & 5 \\
$J_{C, M}$ & cell-matrix adhesive strength & 2.5 \\
$\mu$ & haptotaxis strength & 5 \\
$\lambda_{p}$ & on-rate constant of ECM protein decay & $1.3 \cdot 10^{-4}\left[\mathrm{~s}^{-1}\right]$ \\
$\chi_{p}$ & on-rate constant of ECM protein degradation & $3\left[\mathrm{~h}^{-1}\right]$ \\
$p_{e x t, 0}$ & initial extracellular level of ECM protein & $4[\mu \mathrm{M}]$ \\
$D_{m}$ & diffusion constant of MMPs & $5{ }^{-4}\left[\mu \mathrm{m}^{2} \mathrm{~s}^{-1}\right]$ \\
$\lambda_{m}$ & on-rate constant of MMP decay & $2 \cdot 10^{-3}\left[\mathrm{~s}^{-1}\right]$ \\
$\pi_{m}$ & on-rate constant of MMP production & $5 \cdot 10^{-3}\left[\mathrm{~s}^{-1}\right]$ \\
\hline \hline
\end{tabular}

\subsection{Results}

The simulation domain $\Omega$ represents a $1 \mathrm{~mm}$-size section of tissue. One MCS is set to correspond to 20 sec: the overall simulations stop after $15000 \mathrm{MCS}$, so that they reproduce a time-lapse of nearly 4 days. The initial condition consists of a layer of tumor cells, which is consistent with a spatially extended cancer mass invading from an epithelial cell lining down its basement membrane into the surrounding stroma.

The proposed computational setting allows to capture finger formation and extension from the edge of the tumor mass. As reproduced in Figure 2, the overall process consists of different phases: initially, the more external malignant cells, due to the reduced importance of cell-cell adhesion w.r.t. cell-matrix adhesion and to the haptotactic force, spread in the surrounding tissue as a front of little dissociated islands. Such cell clusters go on increasing in size, due to cell proliferations, and then come in contact with the main tumor mass by short and thick (4-5 cell-wide) fingers. The increased cellular density, in turn, enforces cell-cell adhesive interactions, which balance the effect of the haptotaxis: the formed fingers therefore do not break and stabilize. Finally, the emerged finger structures are able to elongate across the extracellular matrix as a consequence of the simultaneous action of further cell divisions and of the directional haptotactic movement.

We next characterize tumor patterns emerging for different strength of cell-cell adhesive interactions. As briefly sketched in the previous section, the value of the parameter $J_{C, C}$ has a clear biological relevance as, at the molecular level, it gives a measure of the expression and the engagement of cadherins. Indeed, its variation is expected to have a substantial impact on the overall invasiveness and morphology of the disease. At a lower value of the parameter (i.e., $J_{C, C}=1<J_{C, M}$, which means higher cell-cell adhesiveness), the tumor remains compact, with also the external cells that clump along the front of the mass, rather than invading significantly the surrounding tissue, see Figure 3 (right panel). This phenomenology is due to the fact that cell-cell adhesive interactions are too strong to be overcome by the other forces experienced by malignant individuals (i.e., haptotaxis and cell-matrix adhesion) and therefore 

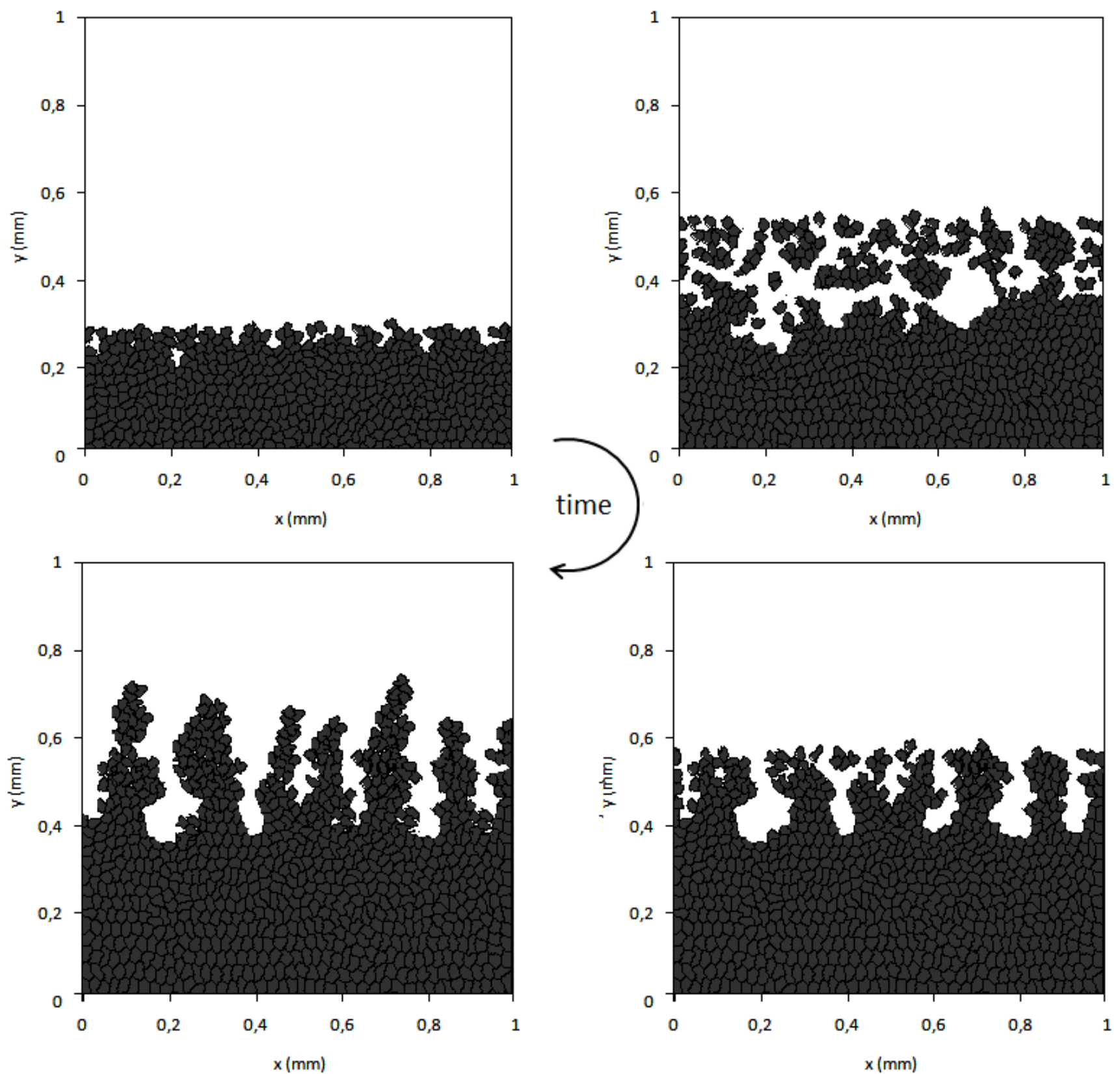

Figure 2. Formation and extension of tumor fingers at the edge of a malignant layer.

stabilize tumor morphology. At a large enough values of $J_{C, C}$ (i.e., $\left.=10\right)$, the tumor instead expands and invades deeper: the external cells quickly lose contacts, dissociate, move from their original site and start wandering in the close proximity, displaying an evident ability to scatter in the surrounding tissue. A repulsion occurs also among individuals within the main body of the mass. The subsequent formation of islands of free matrix within the the tumor, which are then only partially filled in again, increases the overall bias toward invasion, as reproduced in Figure 3 (left panel). The dramatic downregulation of cell-cell adhesiveness indeed induces a sort of mesenchymal transition able to overcome the physiological control of the mechanism of contact-inhibition of cell locomotion. 

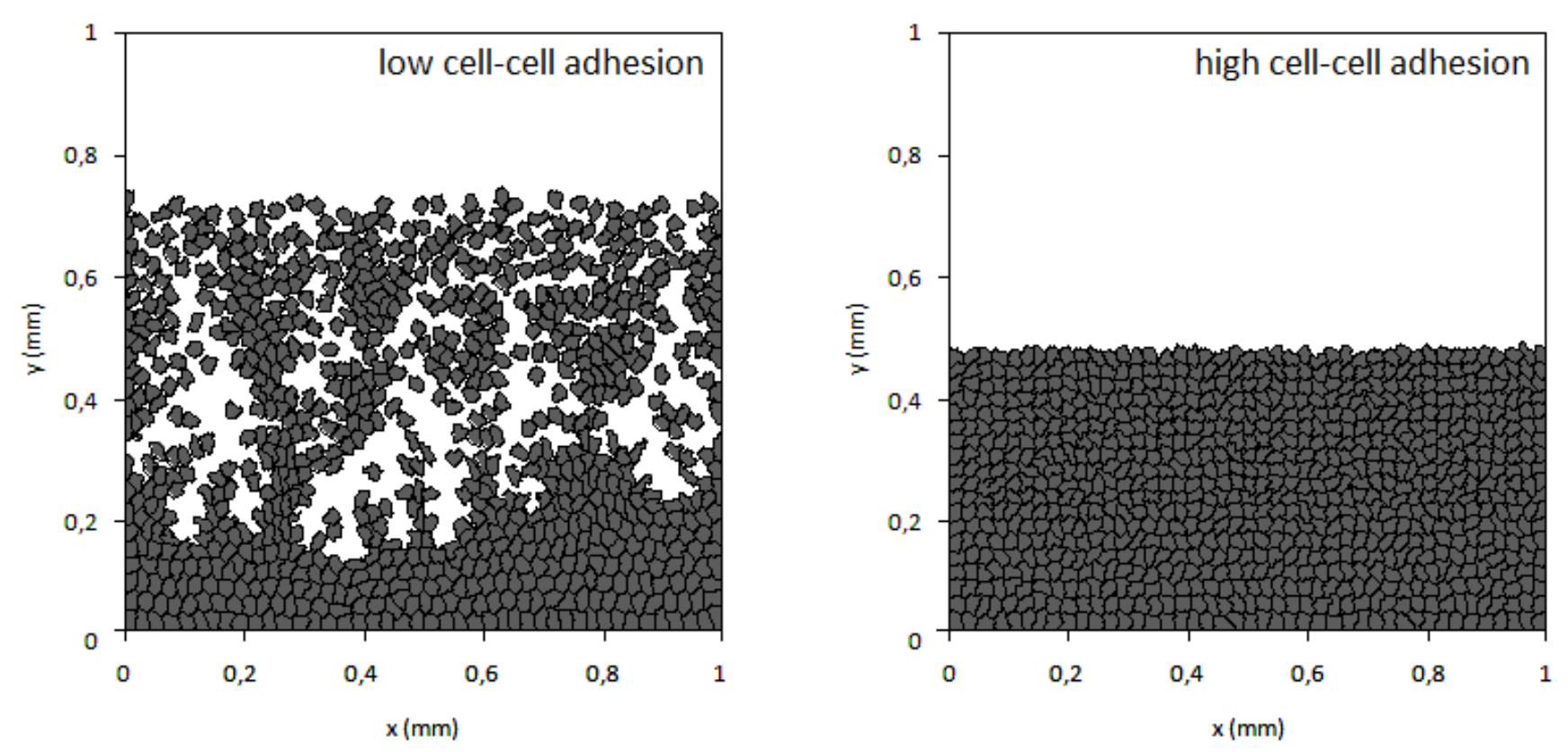

Figure 3. Resulting morphologies of the microinvasive tumor mass for different intercellular adhesive interactions (i.e., given by parameter $\left.J_{C, C}\right)$.

\section{Measure theoretic model}

The measure theoretic-based framework for reproducing tumor fingering is based on the approach presented in $[23,24]$. The basic idea is that by representing cellular aggregates in terms of abstract measures it is possible to choose only a posteriori the scale of representation of the system, i.e., miscroscopic/discrete or macroscopic/continuous. This way, we are allowed to describe each cell population forming a tumor mass at the most suitable scale, i.e., according to its specific biological phenotype. With respect to the CPM presented in the previous Section, the measure-based mathematical environment is again hybrid but no longer nested: continuous and discrete approaches in fact coexist, but they are employed to describe different subsystems characteristic of cellular level, i.e., different cell clones.

\subsection{Model description}

We consider a biological system formed by $P \geq 1$ cell populations, each of them with a finite and constant number of individuals $N_{p}$ (i.e., in order to focus on cell motion, we are assuming that cells do not undergo mitosis or apoptosis). The position at time $t \geq 0$ of a generic representative cell of population $p$, hereafter called test cell, is described by means of a random variable $\mathbf{X}_{p, t}$, which takes values in the measurable space $\left(\mathbb{R}^{n}, \mathcal{B}\left(\mathbb{R}^{n}\right)\right)$, with $n=1,2,3$ for physical reasons. It means that, once an initial position $\mathbf{X}_{p, 0}=\mathbf{x}_{0} \in \mathbb{R}^{n}$ is assigned, the mapping $t \mapsto \mathbf{X}_{p, t}\left(\mathbf{x}_{0}\right)$ represents the trajectory of the cell placed in $\mathbf{x}_{0}$ at the initial time $t=0$. In order to reduce the complexity of the problem, we assume that the cells belonging to each population are indistinguishable, i.e., if we switch the initial position of two cells of the same population, the overall dynamics observed at future instants $t>0$ is the same. Then, the total mass of population $p$ is represented by a Radon positive measure $\mu_{p, t}$, that we assume to be defined on the Borel $\sigma$-algebra $\mathcal{B}\left(\mathbb{R}^{n}\right)$, i.e., for any measurable set $E \in \mathcal{B}\left(\mathbb{R}^{n}\right)$, the value $\mu_{p, t}(E) \geq 0$ measures therefore the mass of cells located in $E$ at time $t \geq 0$. Specifically, assuming that cells can not undergo mitosis or apoptosis,

$$
\mu_{p, t}\left(\mathbb{R}^{n}\right)=N_{p} \quad \forall t \geq 0,
$$


i.e., the total mass of the system is preserved in time. By assuming an overdamped force-velocity response (as implicitly set also for the CPM), a prototype for the evolution equation of the test cell $\mathbf{X}_{p, t}$ writes as:

$$
\begin{aligned}
\dot{\mathbf{X}}_{p, t} & =\mathbf{v}_{p}^{\text {chem }}\left(t, \mathbf{X}_{p, t}\right)+\mathbf{v}_{p}^{\text {int }}\left(\mathbf{X}_{p, t}\right)= \\
& =\mathbf{v}_{p}^{\text {chem }}\left(\mathbf{X}_{p, t}\right)+\sum_{q=1}^{P} \int_{\mathbb{R}^{n} \backslash\left\{\mathbf{X}_{p, t}\right\}} \mathbf{K}^{p q}\left(\mathbf{X}_{p, t}, \mathbf{y}\right) d \mu_{q, t}(\mathbf{y}),
\end{aligned}
$$

where $\mathbf{v}_{p}^{\text {chem }}$ denotes a cell preferred velocity and $\mathbf{v}_{p}^{\text {int }}$ an interaction-dependent velocity. Specifically, $\mathbf{v}_{p}^{\text {chem }}: \mathbb{R}^{n} \rightarrow \mathbb{R}^{n}$ is a motion field generated either by the spatial distribution of some diffusive chemical that test cell is sensitive to (i.e., chemotaxis) or by the topology of the extracellular environment (i.e., via durotactic or haptotactic mechanisms). On the other hand, the velocity interaction component $\mathbf{v}_{p}^{\text {int }}$ : $\mathbb{R}^{n} \rightarrow \mathbb{R}^{n}$ accounts for intercellular adhesive/repulsive interactions performed by the test cell $\mathbf{X}_{p, t}$ of population $p$ through a scanning of the surrounding mass $\mu_{q, t}$ of each population $q=1, \ldots, P$ : such contributions are defined by the interaction kernels $K^{p q}: \mathbb{R}^{n} \times \mathbb{R}^{n} \rightarrow \mathbb{R}^{n}$.

An evolution equation for the overall mass measure of each cell population $\mu_{p, t}$ can be formally derived from (10). Reminding the reader to [23] for entire procedure, we here simply sketch the idea. Being a physical quantity of cells, the cellular mass of each population has to move coherently with the motion of the component individuals, i.e., $\mu_{p, t}$ is transported by $\mathbf{X}_{p, t}$. Then, regarding the mapping $t \mapsto \mu_{p, t}$ as a curve in the space of distributions, one can compute the time derivative of $\mu_{p, t}$ proceeding like in the Reynolds' theorem and obtain the following weak form of the conservation law for the cellular mass:

$$
\frac{d}{d t}\left\langle\mu_{p, t}, \varphi\right\rangle=\int_{\mathbb{R}^{n}} \nabla \varphi(\mathbf{x}) \cdot\left(\mathbf{v}_{p}^{\text {chem }}(t, \mathbf{x})+\mathbf{v}_{p}^{\text {int }}(t, \mathbf{x})\right) d \mu_{p, t}(\mathbf{x})
$$

where $\varphi$ is a test function in the the Banach space $C_{c}^{\infty}\left(\mathbb{R}^{n}\right)$ and $\langle\cdot, \cdot\rangle$ denotes the duality pairing in $C_{c}^{\infty}\left(\mathbb{R}^{n}\right)$. After defining the transport velocity

$$
\mathbf{v}_{p, t}(\mathbf{x}):=\mathbf{v}_{p}^{\text {chem }}(t, \mathbf{x})+\mathbf{v}_{p}^{\text {int }}(t, \mathbf{x})
$$

from Equation (11) it is finally possible to obtain the following strong formulation of the problem:

$$
\left\{\begin{array}{l}
\partial_{t} \mu_{p, t}+\nabla \cdot\left(\mu_{p, t} \mathbf{v}_{p, t}\right)=0, \quad p=1, \ldots, P \\
\mathbf{v}_{p, t}(\mathbf{x})=\mathbf{v}_{p}^{\operatorname{chem}}(\mathbf{x})+\sum_{q=1}^{P} \int_{\mathbb{R}^{n} \backslash\{\mathbf{x}\}} \mathbf{K}^{p q}(\mathbf{x}, \mathbf{y}) d \mu_{q, t}(\mathbf{y}) .
\end{array}\right.
$$

\section{A two population tumor system}

As known from the experimental literature, a solid tumor can be distinguished at least in two cell phenotypes: an external set of few differentiated, aggressive, highly metabolic, individuals and an internal, quiescent and undifferentiated cellular mass. Indeed, in order to formalize a proper mathematical description of the fingering infiltration of a solid tumor with the measure theoretic-based approach, it is straightforward to consider a two-population system $(P=2)$. On one hand, the undifferentiated central cell cluster (i.e., population $p=1$ ) can be represented as a distributed group with a low level of detail by assuming that the corresponding mass measure is absolutely continuous with respect to the $n$-dimensional Lebesgue measure $\mathcal{L}^{n}$ :

$$
\mu_{1, t}(E)=\int_{E} \rho_{t}(\mathbf{y}) d \mathbf{y} \quad \forall E \in \mathcal{B}\left(\mathbb{R}^{n}\right) .
$$

$\rho_{t}(\cdot):[0, T] \times \mathbb{R}^{n} \rightarrow[0,+\infty), T>0$ being a certain final time (possibly $+\infty$ ), is a numerical space density at time $t$, whose existence is asserted by the Radon-Nikodym's Theorem. On the other hand, the 
differentiated cells can be individually described by using an atomic mass measure, constituted of a sum of Dirac masses centered in each cell position as,

$$
\mu_{2, t}(E)=\sum_{i=1}^{N_{2}} \delta_{\mathbf{x}_{t}^{i}}(E) \quad \forall E \in \mathcal{B}\left(\mathbb{R}^{n}\right),
$$

where the $\left\{\mathbf{x}_{t}^{i}\right\}_{i=1}^{N_{2}}$ are the differentiated cell positions at time $t$.

The mathematical structure (13) therefore specifies in a system of coupled discrete-continuous equations, which consists of a conservation equation for $\rho_{t}$ and of a set of ODEs for the $\mathbf{x}_{t}^{i}, i=1, \ldots, N_{2}$ :

$$
\left\{\begin{array}{l}
\partial_{t} \rho_{t}(\mathbf{x})+\nabla \cdot\left(\rho_{t}(\mathbf{x}) \mathbf{v}_{1, t}(\mathbf{x})\right)=0 \\
\dot{\mathbf{x}}_{t}^{i}=\mathbf{v}_{2, t}\left(\mathbf{x}_{t}^{i}\right) \quad i=1, \ldots, N_{2}
\end{array}\right.
$$

where the velocity fields of the two populations are defined as follows:

$$
\left\{\begin{array}{l}
\mathbf{v}_{1, t}(\mathbf{x})=\int_{\mathbb{R}^{n}} K^{11}(\mathbf{x}, \mathbf{y}) \rho_{t}(\mathbf{y}) d \mathbf{y}+\sum_{j=1}^{N_{2}} K^{12}\left(\mathbf{x}, \mathbf{x}_{t}^{j}\right), \\
\mathbf{v}_{2, t}\left(\mathbf{x}_{t}^{i}\right)=\mathbf{v}_{2}^{\text {chem }}\left(t, \mathbf{x}_{t}^{i}\right)+\int_{\mathbb{R}^{n}} K^{21}\left(\mathbf{x}_{t}^{i}, \mathbf{y}\right) \rho_{t}(\mathbf{y}) d \mathbf{y}+\sum_{\substack{j=1 \\
j \neq i}}^{N_{2}} K^{22}\left(\mathbf{x}_{t}^{i}, \mathbf{x}_{t}^{j}\right) .
\end{array}\right.
$$

In particular, we are assuming that only the differentiated cells, which typically express surface receptors, are sensible to a chemical motility factor, as

$$
\mathbf{v}_{2}^{\text {chem }}(t, \mathbf{x})=k_{0} \nabla c(t, \mathbf{x})
$$

where $k_{0}$ is a chemotactic strength and $c$ is the chemical spatial distribution which evolves in time according to a standard reaction-diffusion equation:

$$
\partial_{t} c(t, \mathbf{x})=D \Delta c(t, \mathbf{x})-\frac{c(t, \mathbf{x})}{\tau}
$$

where $D$ is the constant and homogeneous diffusion coefficient and $1 / \tau$ the decay rate. Finally, the hypothesis of isotropic adhesive/repulsive cell interactions, along with the idea that they are respectively directly/inversely proportional to the distance between interacting individuals, leads to $K^{p q}(\mathbf{x}, \mathbf{y})=$ $\mathcal{K}^{p q}(\mathbf{y}-\mathbf{x})$. Specifically, defining the interaction radial vector $\mathbf{r}:=(\mathbf{y}-\mathbf{x})$, the kernels are set to have the following form:

$$
\mathcal{K}^{p q}(\mathbf{r})= \begin{cases}-2 \frac{F_{R}^{p q}}{R_{R}^{p q}} \mathbf{r}, & \text { if }|\mathbf{r}|<\frac{R_{R}^{p q}}{2} \\ \left(2 \frac{F_{R}^{p q}}{R_{R}^{p q}}|\mathbf{r}|-2 F_{R}^{p q}\right) \frac{\mathbf{r}}{|\mathbf{r}|}, & \text { if } \frac{R_{R}^{p q}}{2} \leq|\mathbf{r}|<R_{R}^{p q} \\ -4 F_{A}^{p q} \frac{\left(|\mathbf{r}|-R_{R}^{p q}\right)\left(|\mathbf{r}|-R_{A}^{p q}\right)}{\left(R_{R}^{p q}-R_{A}^{p q}\right)^{2}} \frac{\mathbf{r}}{|\mathbf{r}|}, & \text { if } R_{R}^{p q} \leq|\mathbf{r}|<R_{A}^{p q} \\ 0, & \text { otherwise }\end{cases}
$$

where $R_{R}^{p q}, R_{A}^{p q}>0$ are the repulsive and adhesive radii and $F_{R}^{p q}, F_{A}^{p q}>0$ the corresponding interaction strengths, respectively. 


\subsection{Results}

We deal with a two-dimensional $(n=2)$ square bounded domain $\Omega \subset \mathbb{R}^{2}$, which is set to represent a $900 \times 900 \mu \mathrm{m}^{2}$ section of an in vivo tumor disease, typically used for in vitro biological/biomedical assays. The computational time step $\Delta t$ is assumed to correspond to approximately 10 seconds. Simulations are typically stopped after $T=3600 \Delta t$, so that they reproduce a time-lapse of nearly 12 hours. The numerical simulations are produced by a modified version of the numerical scheme developed in [25] (see also [26-28] for error and convergence analysis), specifically designed for integrating the evolution of a diffusing substance within the basic measure-theoretic framework. The initial configuration of the system consists of $N_{2}=4$ differentiated tumor cells distributed on the edge of a homogeneous and round malignant colony of $N_{1}=100$ inactivated individuals, as shown in the top left panel of Figure 4 . In particular, $\rho_{0}(x)=100 /\left(\pi R_{0}^{2}\right)$ inside the ball $B_{R_{0}} \subset \Omega$ centered in the middle of the domain with radius $R_{0}=100 \mu \mathrm{m}$ and $\rho_{0}(x)=0$ otherwise.

The microenvironmental chemical is assumed to diffuse and decay within the entire domain. In particular, referring to (19), we set $D=10 \mu \mathrm{m}^{2} \mathrm{~s}^{-1}$ and $\tau=5000 \mathrm{~s}$ : these are typical values for some important growth factors, such as many isoforms of the Vascular Endothelial Growth Factor (VEGF). Assuming a constant production of chemical along the entire boundary of the domain we prescribe $c=0.25 \mu \mathrm{Ms}^{-1}$ on $\partial \Omega$ and set the chemotactic strength $k_{0}=500 \mu \mathrm{m}^{2}(\mu \mathrm{Ms})^{-1}$.

The parameters introduced in the interaction kernels (20), describing the biophysical properties of the cells, can be evaluated consistently with biological considerations. Dealing with two populations that are clones of the same cell line (i.e., population 2 is the activated counterpart of population 1), we can consistently take $R_{R}^{p q}=R_{R}=20 \mu \mathrm{m}$ (that is the average size of most tumor cells) and $F_{R}^{p q}=F_{R}=$ $1 \mu \mathrm{ms}^{-1}$ for all $p, q=1,2$. Conversely, as cell activation increases the maximal extension of cell filopods and the quantities of expressed cell adhesion molecules (CAMs), we set

$$
\mathbf{R}_{A}=\left(\begin{array}{cc}
30 & 45 \\
45 & 60
\end{array}\right) \mu \mathrm{m}, \quad \mathbf{F}_{A}=\left(\begin{array}{cc}
0.04 & 0.203 \\
0.0038 & 0.00025
\end{array}\right) \mu \mathrm{ms}^{-1}
$$

The value of $F_{A}^{11}$ assures that population 1 reaches a sort of inner equilibrium, i.e., the continuous colony does not undergo a dramatic scatter or an unrealistic collapse (see [23,24] for a detailed analysis). Moreover, this set of parameters allows for a consistent chemotactic migration of the activated cells, as the heterotypic adhesive forces $F_{A}^{12}$ and $F_{A}^{21}$ are not strong enough to cause their absorption within the continuous mass. Further, the asymmetry of $\mathbf{F}_{A}$ is due to the different scales chosen to represent the two populations (for readers convenience, we underline that $F_{A}^{p q}$ defines the adhesive strength of a test cell of population $p$ in the interaction with a test cell of population $q$ ).

As shown in Figure 4, the round tumor initially reorganizes toward a configuration, which results stable until the chemical diffusive front reaches the colony. Subsequently, the activated malignant cells react to the chemical stimuli: then, four cellular fingers emerge and fully develop from the undifferentiated aggregate besides each discrete moving individual. The first difference from CPM is that we do not observe any front of dissociated islands but a continuous elongation of cellular tongues from main tumor mass. Further, we recall that the measure-based model is obtained under the assumption that the whole mass is preserved in time, i.e., cell proliferation is not included in the model. Thus, in this case the finger formation is uniquely due to the heterotypic interacting term, i.e., it is the result of the differentiation and the adhesive interactions between leader/activated cells and follower/quiescent individuals (determined by the proper values of $F_{A}^{12}$ and $F_{A}^{21}$ which, at molecular level, give the measure of the expression and engagement of cadherins between the activated cells and the quiescent cells).

In this respect, $F_{A}^{12}$ and $F_{A}^{21}$ are the counterpart of the CPM parameter $J_{C, C}$. Indeed, for the sake of completeness, we investigate how the fingering patterning is affected by variations of $F_{A}^{12}$ and $F_{A}^{21}$. As represented in Figure 5, both a decrement and an increment of two orders of magnitude in the values of the heterotypic adhesive forces dramatically inhibit the process of fingering. In particular, at low heterotypic adhesive forces $\left(F_{A}^{12}=0.0203 \mu \mathrm{ms}^{-1}\right.$ and $\left.F_{A}^{21}=0.000038 \mu \mathrm{ms}^{-1}\right)$, we observe the scattering of the differentiated cells. The rationale is that the repulsive heterotypic interactions, as long 

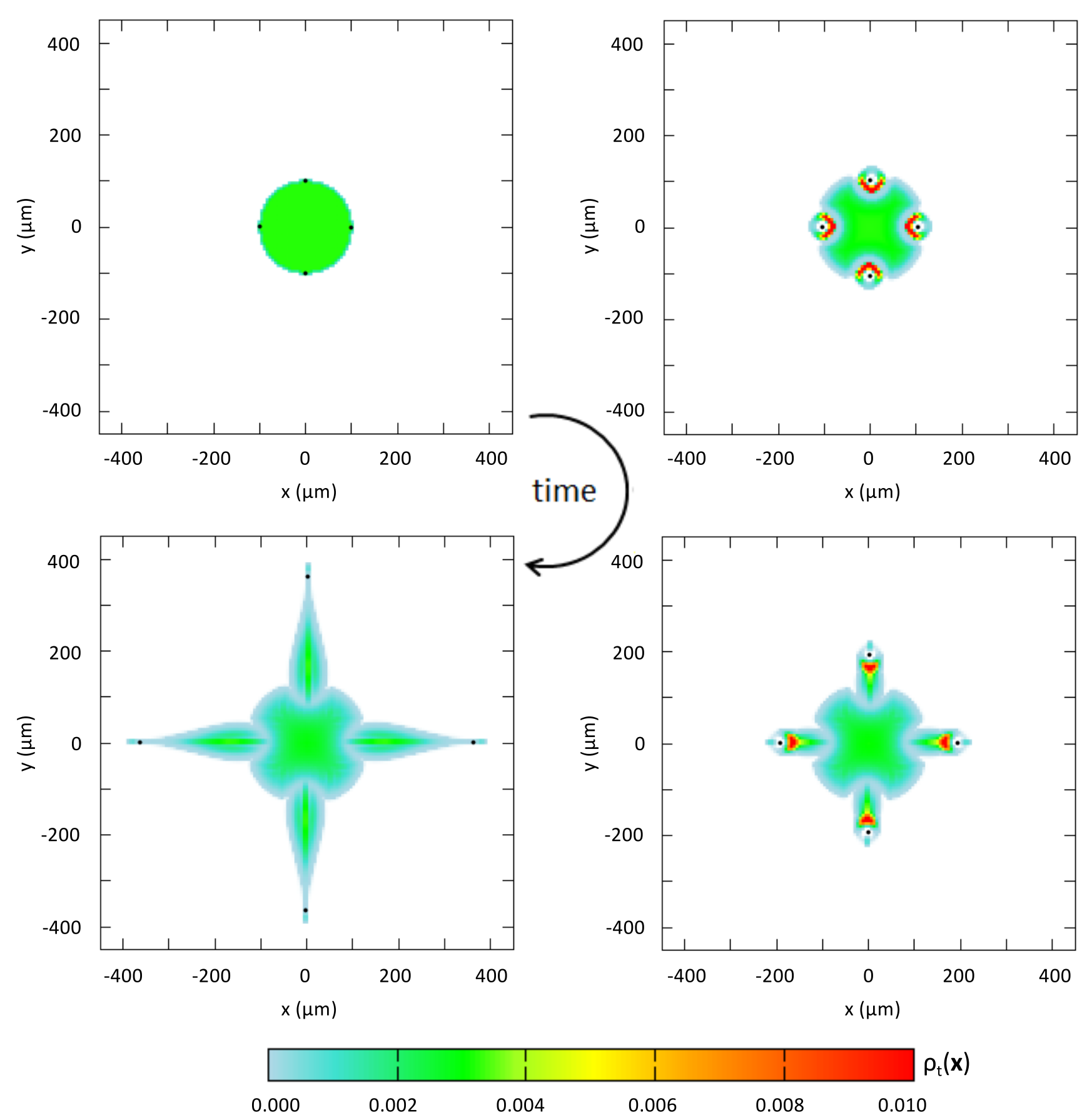

Figure 4. Fingering process resulting in a two-population tumors.

as the chemotactic force, prevails and results in a quick detachment of the discrete individuals from the continuous spheroid. On the other hand, at high heterotypic adhesive forces $\left(F_{A}^{12}=20.3 \mu \mathrm{ms}^{-1}\right.$ and $F_{A}^{21}=0.38 \mu \mathrm{ms}^{-1}$ ), parts of the quiescent population separate from the original colony and form small continuous clusters around each activated cell. The remaining quiescent tumor mass instead remains in the initial position, undergoing only an inner reorganization.

Finally, for a more informative comparison of the numerical results, we simulate with the measuretheoretic model the same tumor configuration considered in Figure 2. As shown in Figure 6 a rectangular layer of undifferentiated cells is indeed placed at the bottom part of a two-dimensional bounded domain 

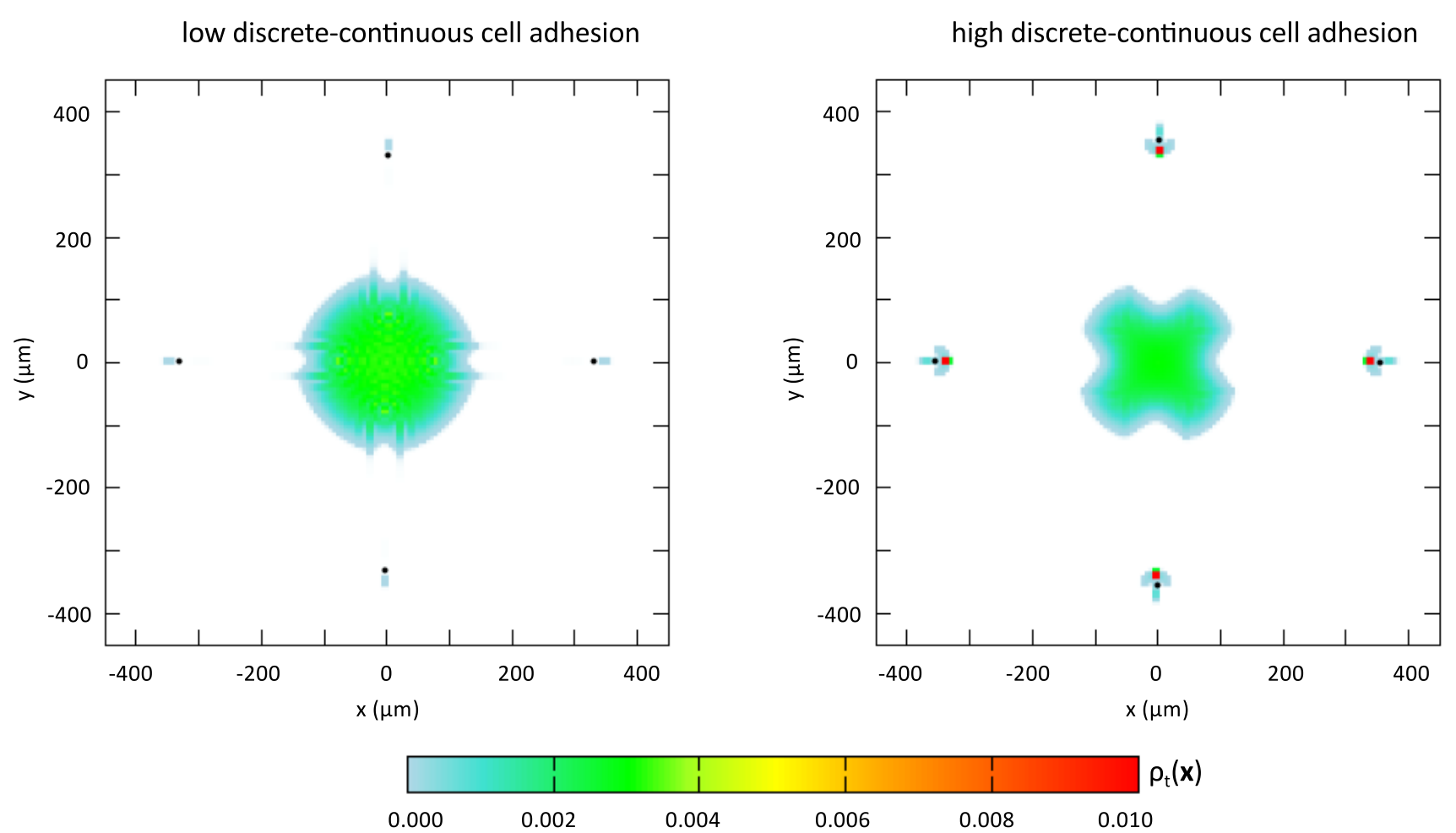

Figure 5. Resulting morphologies of the two-population tumor for different values of the heterotypic adhesive interactions (i.e., given by the parameters $F_{A}^{12}$ and $F_{A}^{21}$ ).

$\Omega \subset \mathbb{R}^{2}$, which is set to represent a $1 \mathrm{~mm}^{2}$ section of a tissue. In particular, $N_{1}=900$ inactivated individuals, which correspond to the initial density $\rho_{0}(x)=0.003 \mu \mathrm{m}^{2}$ inside the subdomain $[0 ; 1000] \times$ $[0 ; 300] \mu \mathrm{m}^{2} . N_{2}=6$ differentiated individuals are then initially seeded at the edge of the malignant mass: they are attracted upwards by a diffusing chemical, which is produced only at the top border of the domain. The parameters regarding both the chemical substance (i.e., diffusion, decay and production coefficients) and the cell populations (i.e., homotypic and heterotypic adhesiveness and interaction radii and chemotactic strength for the activated cells) are the same as in the case of the reference simulation presented in Figure 4. As it is possible to see from the corresponding images in Figure 6, the mass of undifferentiated malignant cells remains almost quiescent. Then, buds of macroscopically-described individuals emerge and extend behind the activated cells, that start migrating upon chemical gradients. At the end of the observation time, coherently with the simulation presented in Figure 4, quite long and thin tumor tongues have invaded the tissue. Such a finger pattern is different from the one captured by the CPM in Figure 2, where the malignant branches are thicker. This discrepancy is due to the different mechanisms underlying finger formation, as previously commented.

\section{Conclusions}

Solid tumor invasion is driven by the complex interplay of molecular- and cellular-scale dynamics of genetically damaged cells, that are able to survive and evolve even under extreme conditions, e.g., hypoxia and acidosis [29]. In particular, the aggressiveness of a cancer mass is mainly affected by altered biophysical characteristics of malignant cells, such as their enhanced motility and metabolism and the downregulation of the intercellular adhesion, as well as by the aggressive interactions with the local microenvironment, such as the increased consumption of available nutrients and degradation of ECM proteins. A significant indicator of the invasiveness of a disease is represented by the morphological instability of its edge. In this respect, many malignancies are characterized by unstable fingered interfaces, which typically result 

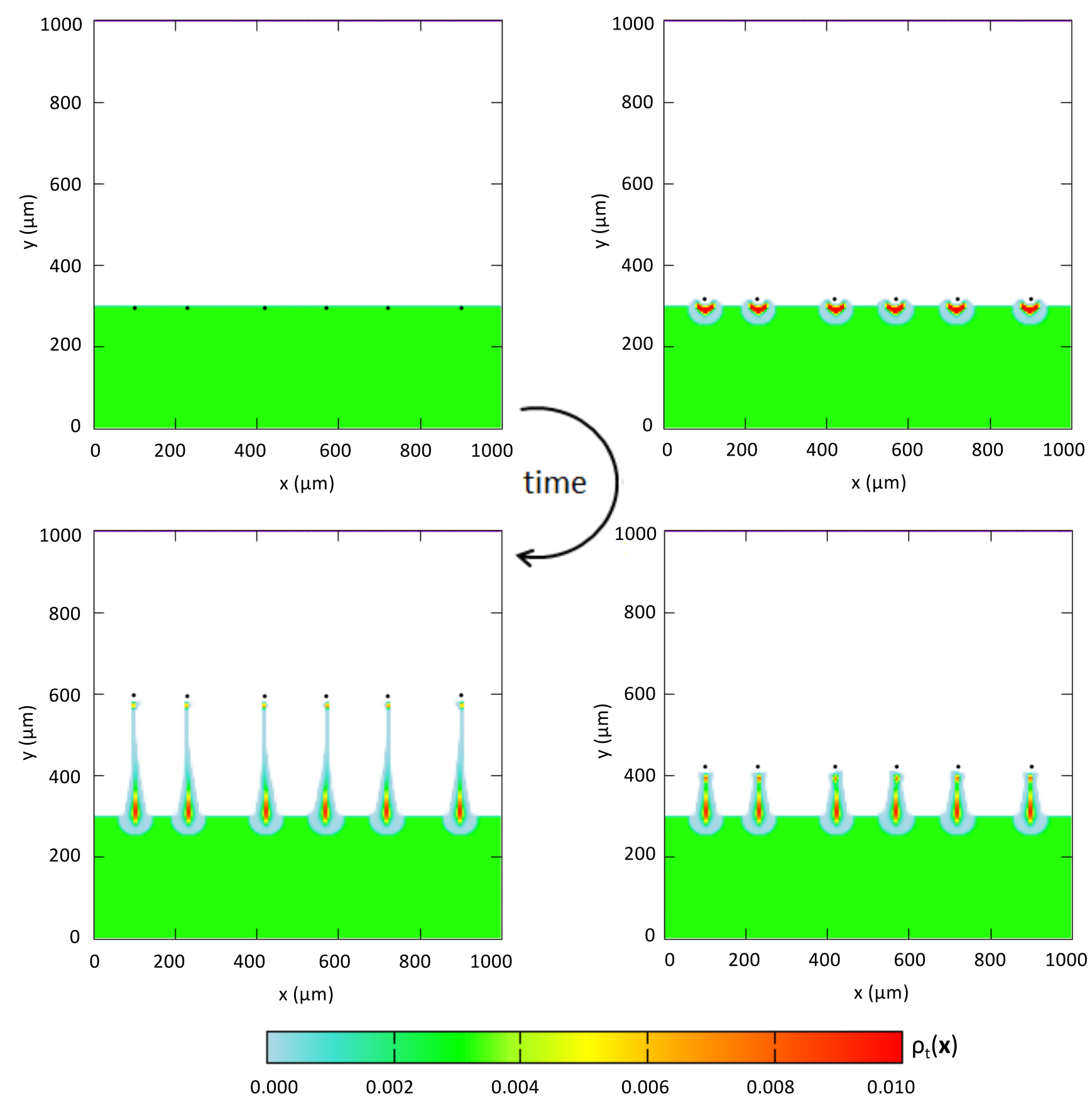

Figure 6. Tumor fingers emerging from a malignant cell monolayer initially placed at the bottom part of the domain. Six differentiated cells are placed at the edge of the tumor mass, i.e., at locations $(100 \mu \mathrm{m} ; 295 \mu \mathrm{m}),(230 \mu \mathrm{m} ; 295 \mu \mathrm{m}),(420$ $\mu \mathrm{m} ; 295 \mu \mathrm{m}),(570 \mu \mathrm{m} ; 295 \mu \mathrm{m}),(720 \mu \mathrm{m} ; 295 \mu \mathrm{m})$, and $(900 \mu \mathrm{m} ; 295 \mu \mathrm{m})$. The chemical substance is produced only at the top border of the domain. All the model parameters are the same as in the simulation reproduced in Figure 4.

in an enhanced severity of the tumor.

In this work, we have presented two different multiscale hybrid approaches, both able to reproduce selected features of the phenomenon of tumor fingering. On one side, the proposed version of the cellular Potts model nests a continuous approximation for molecular variables within an individual-based description of the single malignant cells forming the tumor mass. As a result, the formation and extension of multicellular fingers is due to the conjunct activity of cell proliferation and adhesion and of cell haptotactic movement, that is facilitated by the digestion of non-soluble matrix proteins. On the other side, 
the described measure theoretic approach interfaces a microscopic/discrete representation of the more external and highly metabolic tumor cells and a continuous approximation for the internal quiescent individuals. In this case, finger extension is the consequence of the chemotactic migration of the external cells, which are followed (due to adhesive interactions) by tongues of internal individuals.

Similarities and differences between the two models. Both computational environments shed light on the critical role played by cell-cell adhesion and by taxis-like migration in determining the invasive morphology of the tumor. However, the dynamics underlying the fingering process are quite different. In fact, in the CPM, the multicellular tongues form as a consequence of the contact between scattered island of external cells and the rest of the malignant mass. On the opposite, in the measure-based method, they extend from the tumor edge. Moreover, the two approaches differs for the inclusion/neglection both of cell proliferation and of cell differentiated behavior and for the time-scale of the process, as the CPM deals with days while the measure model with hours. Such a last difference is mainly due to the extension of the tumor represented in the simulations: the lesion accounted in the CPM is much larger and therefore multicellular fingers take much more time to stabilize and grow.

Comparison with pertinent literature. The phenomenon of fingering has been captured by several modeling approaches. First, in [17], a suitable version of the cellular Potts model reproduces different morphologies of tumor invasion fronts. In particular, fingering phenomena are observed only when cell proliferation is not taken into account: otherwise, the authors observe that the lesion invades the ECM as a compact solid mass. In [30], finger extensions are reproduced by a cellular automaton model of tumor growth, which takes into account malignant cell motility, proliferation and death, intercellular adhesion, autocrine and paracrine growth stimulation and stromal destruction. In this work, finger fractal dimensionality is related to selected biophysical determinants of tumor and stromal cells. However, the authors are not able to establish a clear relation between the finger dimensions and the overall biological behavior of the tumor. For instance, some growth properties considered to be associated with an increased degree of the disease malignancy influence fractal dimensionality in opposite directions. In [31], fingering phenomena are instead captured by a diffuse interface continuum model of multispecies tumor growth. The simulations presented therein show that the tumor nonlinearly evolves with a branched-shape configuration. In particular, such a complex structure arises from a diffusional instability, which leads to the creation of buds of elongating fingers. The instability is fundamental for the overall tumor development, as it enables the malignant mass to increase the exposure to nutrient and therefore to overcome the diffusional limitations of growth. With this mechanism, the tumor has in principle the potential to grow indefinitely, as the instability repeats itself on the buds and on the resulting fingers. Finally, in [32], a hybrid discrete-continuum (HDC) model, in which cells are treated as discrete stochastic variables and the microenvironmental parameters as deterministic concentrations, analyzes tumor morphology and phenotypic evolution under different pressure conditions. In particular, invasive masses characterized by fingering margins, which are dominated by few clones with aggressive traits, are observed in harsh conditions of the tumor microenvironment (e.g., hypoxia).

\section{References}

1. B. Alberts, A. Johnson, J. Lewis, M. Raff, K. Roberts, and P. Walter, Molecular Biology of the Cell, 4th ed. Garland Science, 2002.

2. H. Osada and T. Takahashi, Genetic alterations of multiple tumor suppressors and oncogenes in the carcinogenesis and progression of lung cancer, Oncogene, vol. 21, pp. 7421-7434, 2002.

3. W. Mueller-Klieser, Tumor biology and experimental therapeutics, Crit. Rev. Oncol. Hematol., vol. 36, pp. 123-139, 2002.

4. P. Vaupel and M.Hockel, Blood supply, oxygenation status and metabolic micromilieu of breast cancers: characterization and therapeutic relevance (review), Int. J. Oncol., vol. 17, pp. 869-879, 2000.

5. J. M. Brown, Tumor microenvironment and the response to anticancer therapy, Cancer Biol. Ther., 


\section{Scianna, A. Colombi}

vol. 1, pp. 453-458, 2002.

6. S. S. Cross, Fractals in pathology, J. Pathol., vol. 182, pp. 1-8, 1997.

7. G. Landini, Y. Hirayama, T. J. Li, and M. Kitano, Increased fractal complexity of the epithelial connective tissue interface in the tongue of 4nq0-treated rats, Pathol. Res. Pract., vol. 196, pp. 251$258,2000$.

8. A. Balter, R. M. H. Merks, N. J. Poplawski, M. Swat, and A. J. Glazier, The Glazier-Graner-Hogeweg model: extensions, future directions, and opportunities for further study, in Single-Cell-Based Models in Biology and Medicine (A. R. A. Anderson, M. A. J. Chaplain, and K. A. Rejniak, eds.), Mathematics and Biosciences in Interactions, pp. 151-167, Birkaüser, 2007.

9. J. A. Glazier and F. Graner, Simulation of the differential adhesion driven rearrangement of biological cells, Phys. Rev. E, vol. 47, pp. 2128-2158, 1993.

10. J. A. Glazier, A. Balter, and N. J. Poplawski, Magnetization to morphogenesis: a brief history of the Glazier-Graner-Hogeweg model, in Single-Cell-Based Models in Biology and Medicine (A. R. A. Anderson, M. A. J. Chaplain, and K. A. Rejniak, eds.), Mathematics and Biosciences in Interactions, pp. 79-106, Birkaüser, 2007.

11. F. Graner and J. A. Glazier, Simulation of biological cell sorting using a two dimensional extended Potts model, Phys. Rev. Lett., vol. 69, pp. 2013-2017, 1992.

12. M. Scianna and L. Preziosi, Multiscale developments of the cellular Potts model, Multiscale Model. Simul., vol. 10, pp. 342-382, 2012.

13. E. Ising, Beitrag zur theorie des ferromagnetismus, Z. Physik., vol. 31, p. 253, 1925.

14. R. B. Potts, Some generalized order-disorder transformations, Proc. Camb. Phil. Soc., vol. 48, pp. 106109, 1952.

15. R. M. H. Merks and P. Koolwijk, Modeling morphogenesis in silico and in vitro: Towards quantitative, predictive, cell-based modeling, Math. Model. Nat. Phenom., vol. 4, pp. 149-171, 2009.

16. M. Scianna, L. Munaron, and L. Preziosi, A multiscale hybrid approach for vasculogenesis and related potential blocking therapies, Prog. Biophys. Mol. Biol., vol. 160, pp. 450-462, 2010.

17. S. Turner and J. A. Sherratt, Intercellular adhesion and cancer invasion: A discrete simulation using the extended potts model, J. Theor. Biol., vol. 216, pp. 85-100, 2002.

18. N. Metropolis, A. Rosenbluth, M. N. Rosenbluth, A. H. Teller, and E. Teller, Equation of state calculations by fast computing machines, J. Chem. Phys., vol. 21, pp. 1087-1092, 1953.

19. M. S. Steinberg, Does differential adhesion govern self-assembly processes in histogenesis? equilibrium configurations and the emergence of a hierarchy among populations of embryonic cells, J. Exp. Zool., vol. 173, pp. 395-433, 1970.

20. S. Huang and D. E. Ingber, The structural and mechanical complexity of cell-growth control, Nat. Cell Biol., vol. 1, pp. 131-138, 1999.

21. N. J. Savill and P. Hogeweg, Modelling morphogenesis: From single cells to crawling slugs, J. Theor. Biol., vol. 184, pp. 118-124, 1997.

22. G. Murphy and J. Gavrilovic, Proteolysis and cell migration: Creating a path?, Curr. Opin. Cell Biol., vol. 11, pp. 614-621, 1999.

23. A. Colombi, M. Scianna, and A. Tosin, Differentiated cell behavior: a multiscale approach using measure theory, J. Math. Biol., 2015, in press. doi: 10.1007/s00285-014-0846-z.

24. A. Colombi, M. Scianna, and L. Preziosi, A measure-theoretic model for collective cell migration and aggregation, Math. Model. Nat. Phenom., vol. 1, no. 10, pp. 32-63, 2015.

25. E. Cristiani, B. Piccoli, and A. Tosin, Multiscale modeling of granular flows with application to crowd dynamics, Multiscale Model. Simul., vol. 9, no. 1, pp. 155-182, 2011.

26. B. Piccoli and F. Rossi, Transport equation with nonlocal velocity in Wasserstein spaces: convergence of numerical schemes, Acta Appl. Math., vol. 124, no. 1, pp. 73-105, 2013.

27. B. Piccoli and A. Tosin, Time-evolving measures and macroscopic modeling of pedestrian flow, Arch. Ration. Mech. Anal., vol. 199, no. 3, pp. 707-738, 2011. 
28. A. Tosin and P. Frasca, Existence and approximation of probability measure solutions to models of collective behaviors, Netw. Heterog. Media, vol. 6, no. 3, pp. 561-596, 2011.

29. R. Gatenby, K. Smallbone, P. Maini, F. Rose, J. Averill, R. Nagle, L. Worrall, and R. Gillies, Cellular adaptations to hypoxia and acidosis during somatic evolution of breast cancer, Br. J. Cancer, vol. 97, pp. $646-653,2007$.

30. J. Smolle, Fractal tumor stromal border in a nonequilibrium growth model, Anal. Quant. Cytol. Histol., vol. 20, pp. 7-13, 1998.

31. S. M. Wise, J. S. Lowengrub, H. B. Frieboes, and V. Cristini, Three-dimensional multispecies nonlinear tumor growth-i model and numerical method, Int. J. Oncol., vol. 253, pp. 524-543, 2008.

32. A. R. A. Anderson, A. M. Weaver, P. T. Cummings, and V. Quaranta, Tumor morphology and phenotypic evolution driven by selective pressure from the microenvironment, Cell, vol. 127, no. 5, pp. 905-915, 2006. 